BULL. AUSTRAL. MATH. SOC.

\title{
DECOMPOSITIONS OF GRAPHS OF MODULES \\ OVER SEMISIMPLE RINGS
}

\author{
GRACE ORZECH
}

\begin{abstract}
In this paper we show that an $R$-module graph over a semisimple ring $R$ can be written as a direct sum of graphic submodules that are uniquely determined up to isomorphism type. Moreover, this decomposition enables us to describe the $R$-module graph in graphic terms as a disjoint union of connected components, each of which consists of a complete directed graph on its vertices together with a set of loops at each vertex, determined by the loops at 0 . We also give a graphic version of Maschke's Theorem.
\end{abstract}

\section{Introduction}

In [4] and [5] Ribenboim described a way of endowing an algebraic object with a compatible directed graph structure. For example, an $R$-module graph $M_{\Gamma}$ is a quadruple $M_{\Gamma}=(M, V(M), 0, t)$ where $M$ is an ordinary $R$-module, $V(M)$ is a submodule of $M$, and $0, t: M \rightarrow V(M)$ are $R$-homomorphisms that restrict to the identity on $V(M)$. Thus, $M=\operatorname{ker}(o) \oplus V(M)=\operatorname{ker}(t) \oplus V(M)$. These decompositions are natural in an algebraic sense but unsatisfying categorically because $\operatorname{ker}(0)$ and $\operatorname{ker}(t)$ are submodules which are not $R$-module graphs in their own right.

When $R$ is a field we are dealing with vector space graphs and in

Received 5 January 1984.

Copyright Clearance Centre, Inc. Serial-fee code: 0004-9727/84 $\$ \mathrm{~A} 2.00+0.00$. 
[3] we have studied decompositions of $M_{\Gamma}$ into indecomposable $R$-subspaces. An analogous decomposition is possible when $R$ is a semisimple ring in the usual (nongraphic) sense.

Before launching into the study of $R$-module graphs over a semisimple ring, $R$, we recall some observations made in [2] concerning arbitrary $R$-module graphs.

First, an $R$-module graph $M_{\Gamma}$ is a directed graph whose vertices are the elements of $V(M)$ and whose edges are the elements of $E(M)=M \backslash V(M)$. An edge $e$ is directed from o(e) to $t(e)$.

A submodule $M^{\prime}$ of $M$ is called a graphic submodule if $o\left(M^{\prime}\right) \cup t\left(M^{\prime}\right) \subseteq M^{\prime}$.

Let $t(\operatorname{ker}(0))=V_{0}$ be the set of vertices that are graph theoretically adjacent to $0 . C_{0}=\operatorname{ker}(0) \oplus V_{0}$ is a graphic submodule of $M$ and it is the graph theoretic connected component of 0 .

$L_{0}=\operatorname{ker}(0) n \operatorname{ker}(t)$ is a graphic submodule and it consists of the vertex 0 together with all loops at 0 .

When $R$ is semisimple, ordinary $R$-modules are completely reducible in the sense described in [1, Chapter II]. This is not the case for $R$-module graphs, as we shall see.

1. A graphic decomposition of an R-module graph

The decomposition $M=\operatorname{ker}(0) \oplus V(M)$ is module theoretic but not graph theoretic since $\operatorname{ker}(0)$ is not a subgraph of $M_{\Gamma}$ unless $\operatorname{ker}(0)=\operatorname{ker}(t)=L_{0}$. Similarly the decomposition $C_{0}=\operatorname{ker}(0) \oplus V_{0}$ has components which are not graphic.

When $R$ is semisimple we can express $C_{0}$ and $M$ as direct sums of graphic submodules. To do this, first write $\operatorname{ker}(0)=L_{0} \oplus C$. This can be done because $L_{0} \subseteq \operatorname{ker}(0)$ and $\operatorname{ker}(0)$ is completely reducible. The complement $C$ of $L_{0}$ in $\operatorname{ker}(0)$ is not unique, but the next proposition shows that for any choice of $C, V_{0} \approx C$. 
Proposition $\mathbf{1 . 1}$ If $C \subseteq \operatorname{ker}(0)$ and $C$ satisfies $\operatorname{ker}(0)=L_{0} \oplus C$, then $C \approx V_{0}$.

Proof. Since $c \subseteq \operatorname{ker}(0), t(c)$ is in $V_{0}$ for each $c$ in $c$. Thus we can define $\varphi_{C}: C \rightarrow V_{O}$ by

$$
\varphi_{C}(c)=t(c)
$$

If $\varphi_{C}(c)=0$, then $c$ is in $L_{0} \cap C=\{0\}$. Thus $\varphi_{C}$ is one-one. Let $v$ be any element in $V_{0^{*}}$, we can write $v=t(x)$ for some $x$ in ker( 0 ) since $v$ is adjacent to 0 . By hypothesis, $x=\ell+c$ for unique $\ell$ in $L_{0}$ and $c$ in $C$. Thus,

$$
\varphi_{C}(c)=\varphi_{C}(x-\ell)=t(x-\ell)=t(x)-t(\ell)=v .
$$

So, $\varphi_{C}$ is onto.

Given $C$ as in Proposition 1.1, let $K_{0, C}=C \oplus V_{0^{*}}$ The properties of $K_{0, C}$ are summarized in the next proposition.

Proposition $1.3 K_{0, C}$ is a graphic submodule of $M$. Moreover, $K_{0, C}$ is a complete directed graph in the sense that for any ordered pair, $(v, w)$ of vertices, $v \neq w$, from $k_{0, C}$, there is a unique edge $e$ in $K_{0, C}$ satisfying $o(e)=v$ and $t(e)=w$.

Proof. Given any $k$ in $K_{0, C}$, write $k=c+v$ where $c$ is in $C$ and $v$ is in $V_{0}$. Since $t(c)$ is also in $V_{0}, t(k)=t(c)+v$ is in $V_{O^{*}}$ Also, $o(k)=o(c)+o(v)=v$ is in $V_{O^{*}}$. Thus, $t\left(K_{0, C}\right) \cup o\left(K_{0, C}\right) \subseteq v_{0} \subseteq K_{0, C}$, which proves that $K_{0, C}$ is graphic.

Next consider $v \neq w$ in $V_{0}=V\left(K_{0, c}\right)$. From the proof of Proposition 1.1, we know that there exist $c_{v}$ and $c_{w}$ in $c$ such that $v=t\left(c_{v}\right)$ and $w=t\left(c_{w}\right)$. The edge $e=v-c_{v}+c_{w}$ is in $K_{0, C}$ and satisfies

$$
\begin{aligned}
& o(e)=o(v)-o\left(c_{v}\right)+o\left(c_{w}\right)=v, \\
& t(e)=t(v)-t\left(c_{v}\right)+t\left(c_{w}\right)=v-v+w=w .
\end{aligned}
$$


If $e^{\prime}$ in $C$ also satisfies $o\left(e^{\prime}\right)=v$ and $t\left(e^{\prime}\right)=w$, then $e-e^{\prime}$ is in $L_{O} \cap K_{O, C}=\{0\}$. This proves the uniqueness of the edge from $v$ to $w$.

Now let $C$ be such that $\operatorname{ker}(0)=L_{0} \oplus C$. Then

$$
c_{0}=\operatorname{ker}(0) \oplus V_{0}=\left(L_{0} \oplus C\right) \oplus V_{0}=L_{0} \oplus\left(C \oplus V_{0}\right)=L_{0} \oplus K_{0, C}
$$

Thus $C_{0}$, the connected component of 0 , is the direct sum of two graphic submodules, one characterized graphically as loops at 0 and having only one vertex, the other characterized as a complete directed graph on the vertices in the connected component of 0 . While $L_{0}$ is unique, the component $K_{0, C}$ is determined only up to isomorphism.

Since $R$ is semisimple and $V_{0} \subseteq V(M)$, we can write $V(M)=V_{0} \oplus W$. $W$ is graphic because every submodule of $V(M)$ is graphic. Thus,

$$
\begin{aligned}
M & =\operatorname{ker}(0) \oplus V(M) \\
& =\operatorname{ker}(0) \oplus\left(V_{0} \oplus W\right) \\
& =\left(\operatorname{ker}(0) \oplus V_{0}\right) \oplus \mathrm{W} \\
& =C_{0} \oplus \mathrm{W} \\
& =L_{0} \oplus K_{0, C} \oplus W
\end{aligned}
$$

is a way of expressing $M$ as a direct sum of graphic submodules.

It is easy to see that the submodule $W \approx V(M) / V_{0}$ is determined up to isomorphism. It plays a role in helping to describe $M$ as a graph.

PROPOSITION 1.4. As a graph, $M$ is the disjoint union of subgraphs, $C_{w}$, where $C_{w}$ is the connected component of $w$, and $w$ varies over $W$. Moreover, each $c_{w}$ is graph theoretically isomorphic to $c_{0}$

Proof. Let $w_{1}$ and $w_{2}$ be in $W$ and suppose $c_{w_{1}}=c_{w_{2}}$. Then $w_{1}-w_{2}$ is in $C_{0} \cap V(M)$. Thus, $w_{1}-w_{2}$ is in $v_{0}$. But then $0+w_{1}=\left(w_{1}-w_{2}\right)+w_{2}$ and since $V(M)=V_{0} \oplus W$, we have $0=w_{1}-w_{2}$ and $w_{1}=w_{2}$. 
This shows that

$$
M=\underbrace{}_{\substack{\text { (disjoint) } \\ w \text { in } W}} C_{w} .
$$

To see that $C_{0}$ and $C_{w}$ are isomorphic as graphs, define $F: C_{w} \rightarrow M$ by $F(x)=x-w$ and $G: C_{0} \rightarrow M$ by $G(x)=x+w$. First we check that $F\left(C_{w}\right) \subseteq C_{0^{*}}$ Let $v$ be a vertex in $C_{w}$ and $e$ an edge in $C_{w}$. There is a sequence of edges, $e_{1}, \ldots, e_{k}$ in $C_{w}$ with o(e $)^{\prime}=w$, $t\left(e_{i}\right)=o\left(e_{i+1}\right)$ for $i=1, \ldots, k-1$, and $t\left(e_{k}\right)=v$, and there is another sequence, $f_{1}, \ldots, f_{s}$, with $o\left(f_{1}\right)=w, t\left(f_{i}\right)=o\left(f_{i+1}\right)$ for $i=1, \ldots, s-1$, and $t\left(f_{s}\right)=o(e)$. But then, $o\left(e_{1}-w\right)=0$, $t\left(e_{i}-w\right)=o\left(e_{i+1}-w\right)$ for $i=1, \ldots, k-1$, and $t\left(e_{k}-w\right)=v-w=F(v)$. So $F(v)$ is in $C_{0^{*}}$ Also, o $\left(f_{1}-w\right)=0, t\left(f_{i}-w\right)=o\left(f_{i+1}-w\right)$ for $i=1, \ldots, s-1$, and $t\left(f_{s}-w\right)=t\left(f_{s}\right)-w=o(e)-w=o(e-w)=$ o(F(e)). Thus, $F(e)$ is in $C_{0}$.

A similar argument can be used to show that $G\left(C_{0}\right) \subseteq C_{w^{*}}$ since

$$
\begin{aligned}
& G F(x)=G(x-w)=(x-w)+w=x \text { and } \\
& E G(x)=E(x+w)=(x+w)-w=x,
\end{aligned}
$$

$F$ and $G$ are one-one and onto.

To see that $F$ and $G$ preserve graphic structure, note that

$$
\begin{aligned}
& O E(x)=o(x-w)=o(x)-w=F o(x) \text { and } \\
& t E(x)=t(x-w)=t(x)-w=F t(x)
\end{aligned}
$$

and similarly, $O G(x)=G o(x)$ and $t G(x)=G t(x)$.

In accordance with our observations we name the components $L_{0}$, $K_{O, C}$, and $W$ of $M$ as follows:

DEFINITION 1.5. $L_{0}$ is called the $200 p$ component of $M, K_{0, C}$ is called the complete component, and $W$ is called the partition component. 
The loop and partition components of a graphic module $M$ share the property that any ordinary submodule of either one is graphic. $k_{0, C}$ is different. $C$ is a submodule of $K_{0, C}$ but it is not graphic because $t(C)=V_{0}$ but $V_{0} \notin C$ since $C \cap V_{0} \subseteq \operatorname{ker}(0) \cap V_{0}=\{0\}$

\section{Decomposition of $R$-module graphs into indecomposable graphic R-submodules.}

If an $R$-module graph $M_{\Gamma}$ is indecomposable, then it must be comprised entirely of one of its graphic components.

Definition 2.1. An $R$-module graph $M_{\Gamma}$ is called loop type if $M=L_{0}$, complete type if $M=K_{0, C}$, and vertex type if $M=W$.

If $M_{\mathrm{r}}$ is a loop type or vertex type indecomposable $R$-module graph then $M$ must be irreducible as an $R$-module since any nontrivial submodule would be a direct summand and the summands would automatically be graphic. On the other hand, if $M_{\Gamma}$ is a complete type indecomposable, then $M$ is not indecomposable as an $R$-module since $M=C \oplus V_{0}$ with $C \approx V_{0} \neq\{0\}$

Proposition 2.2. A complete type R-module graph $K_{0, C}=C \oplus V_{0}$ is indecomposable if and only if $V_{0}$ is an irreducible R-module.

In order to prove this it will be useful to know the next fact:

Lemma 2.3. If $C \oplus V_{0}$ is a complete type R-module graph and $V$ is any submodule of $V_{0}$, then $K=\varphi_{C}^{-1}(V) \oplus V$ is a graphic submodule (where $\varphi_{C}$ was defined in equation (1.2)).

Proof. Each $k$ in $K$ can be written uniquely as $k=x+v$ where $x$ is in $\varphi_{C}^{-1}(V)$ and $v$ is in $V$. Thus,

$$
\begin{aligned}
& o(k)=o(x)+o(v)=v \text { and } \\
& t(k)=t(x)+t(v)=\varphi_{C}(x)+v .
\end{aligned}
$$

This shows that $o(K) U t(K) \subseteq V=V(K)$. 
Proof (of Proposition 2.2.). First suppose $V_{0}$ is not irreducible. Since $R$ is semisimple, $V_{0}=V_{1} \oplus \ldots \oplus V_{n}$, where each $V_{i}$ is an irreducible $R$-module. Let $K_{i}=\varphi_{C}^{-1}\left(V_{i}\right) \oplus V_{i}$. It is graphic by Lemma 2.3 and given the nature of $\varphi_{C}$, it is clear that $K_{0, C}=K_{1} \oplus \ldots \oplus K_{n}$. That is, $K_{0, C}$ is not indecomposable.

On the other hand, suppose $V_{O}$ is irreducible but $K_{O, C}=G \oplus H$ for some graphic submodules $G$ and $H$. since $V\left(K_{0, C}\right)=V_{0}=V(G) \oplus V(H)$, we may assume that $V(G)=V_{0}$ and $V(H)=\{0\}$. Thus, $H \subseteq L_{0}$ which is $\{0\}$ for a complete type $R$-module. This shows that $K_{0, C}$ is indecomposable.

The next fact is an easy consequence of our understanding of the nature of indecomposable $R$-module graphs and the usual decomposition theorem for modules over semisimple rings (e.g. see [1]).

THEOREM 2.4. If $M_{\Gamma}$ is any R-module graph then

$$
M=L_{01} \oplus \ldots \oplus L_{0 \lambda} \oplus K_{1} \oplus \ldots \oplus K_{\tau} \oplus W_{1} \oplus \ldots \oplus W_{\mu}
$$

where each $L_{0 i}$ is a loop type indecomposable, each $K_{j}$ is a complete type indecomposable, and each $W_{k}$ is a vertex type indecomposable. The numbers $\lambda, \tau$, and $\mu$ are unique and if

$$
M=L_{01}^{\prime} \oplus \ldots \oplus L_{0 \lambda}^{\prime} \oplus K_{1}^{\prime} \oplus \ldots \oplus K_{\tau}^{\prime} \oplus \ldots \oplus W_{1}^{\prime} \oplus \ldots \oplus W_{\mu}^{\prime}
$$

is another decomposition of $M$ into indecomposable graphic submodules, the indices may be chosen so that $L_{0 i} \approx L_{0 i}^{\prime}, K_{j} \approx K_{j}^{\prime}$, and $W_{k} \approx W_{k}^{\prime}$ for $i=1, \ldots, \lambda, j=1, \ldots, \tau$, and $k=1, \ldots, \mu$.

\section{Modules over semisimple group rings.}

In this section we suppose $R=k[G]$ where $k$ is a field of characteristic not dividing the order of G. By Maschke's Theorem, $R$ is known to be a semisimple ring. Each $R$-module graph $M_{\Gamma}$ is also a 
$k$-vector space graph. Observe also that each $g$ in $G$ acts as a graphic $k$-linear operator, $T(g)$, on $M_{\Gamma}$.

The main theorem of this section shows how the graphic vector space structure of $M_{\Gamma}$ influences its graphic module structure. It is a relative of Maschke's Theorem.

THEOREM 3.1. Let $k$ be a fiezd and $G$ a finite group satisfying char $(k) \backslash[G: 1]$. Let $M_{\Gamma}$ be a k[G]-module graph and $M_{\Gamma}^{\prime}$ a graphic submodule of $M_{\Gamma}$. If there is a grophic k-subspace $N$ of $M$ such that $M=M^{\prime} \oplus N$, then there is a graphic k[G]-submodule $N^{\prime}$ of $M$ such that $M=M^{\prime} \oplus N^{\prime}$.

Proof. Let $E: M_{\Gamma}+M_{\Gamma}^{\prime}$ be the projection of $M$ onto $M^{\prime}$ arising from the decomposition $M=M^{\prime} \oplus N . \quad E$ is a graphic linear transformation and so is

$$
F=\frac{1}{[G: 1]} \sum_{g} T(g) E T(g)^{-1}
$$

It is easy to check that for each $g$ in $G, F T(g)=T(g) F$, which means $F$ is actually a graphic $K[G]$-module homomorphism that maps $M_{\Gamma}$ to $M_{\Gamma}^{\prime}$. Also easy to verify is the fact that $F \mid M^{\prime}=i d_{M^{\prime}}$.

Now let $N^{\prime}=\left(i d_{M}-F\right)(M) . \quad N^{\prime}$ is a $k[G]$-submodule of $M$ since $i d_{M}$ and $F$ are $k[G]$-homomorphisms.

Let $s$ be in $\{o, t\}$ and $m$ in $M$. Then

$$
s(m-F(m))=s(m)-s F(m)=s(m)-F(s(m))=\left(i d_{M}-F\right)(s(m)) .
$$

This shows that $o\left(N^{\prime}\right) \cup t\left(N^{\prime}\right) \subseteq N^{\prime}$. Thus, $N^{\prime}$ is a graphic $k[G]$-submodule of $M$ and $F: M_{\Gamma} \rightarrow M_{\Gamma}^{\prime}$ may be interpreted as a projection homomorphism corresponding to the desired decomposition: $M_{\Gamma}=M_{\Gamma}^{\prime} \oplus N_{\Gamma}^{\prime}$. 


\section{References}

[1] C. W. Curtis and I. Reiner, Representation theory of finite groups and associative algebras (Interscience Publishers, 1962.)

[2] G. Orzech, "R-module graphs", preprint.

[3] G. Orzech, "A graph theoretic description of vector spaces with three subspaces", preprint.

[4] P. Ribenboim, "Algebraic structures on graphs", Algebra Universalis, 16 (1983), 105-123.

[5] P. Ribenboim, "Vector space graphs", Nanta Math. 12 (1979), 125-132.

Department of Mathematics and Statistics,

Queen's University,

Kingston K7L $3 \mathrm{~N} 6$,

Canada. 